\title{
HOMOGENEOUS REAL HYPERSURFACES
}

\author{
NANCY K. Stanton
}

Let $M$ be an analytic real hypersurface through the origin in $\mathbf{C}^{n}$. The hypersurface $M$ is called weighted homogeneous if it is locally equivalent, via a biholomorphic map which preserves the origin, to a hypersurface given by an equation of the form

$$
P(z, \bar{z})=0
$$

where $P$ is a polynomial which is homogeneous with respect to a nonisotropic group of dilations (see Section 1). If $M$ is weighted homogeneous and $m$ is the weight of the polynomial $P$ in (0.1), we say $M$ is weighted homogeneous of weight $m$. In this paper, we study the question of how one can tell whether $M$ is weighted homogeneous. Our main result, Theorem 4.1, gives a sufficient condition for $M$ to be weighted homogeneous.

For the case of hypersurfaces in $\mathbf{C}^{2}$ which are rigid in the sense of [BRT] (see Section 4), we proved Theorem 4.1 in $[\mathrm{S} 2, \mathrm{~S} 3]$ (see also [S1]). In [S4] we proved the theorem for holomorphically nondegenerate rigid hypersurfaces in the case that the group of dilations is $\delta_{t}(z)=\left(t z_{1}, \ldots, t z_{n-1}, t^{m} z_{n}\right)$. Unlike our earlier results, Theorem 4.1 does not require that $M$ be rigid and there is no nondegeneracy hypothesis or finite type hypothesis on $M$. However, if $M$ is weighted homogeneous, then it is rigid (see Proposition 4.3).

After some preliminaries in Section 1, we prove some lemmas concerning allowable maps in Section 2, maps which are the identity map modulo terms of higher weight. In Section 3 we show that certain holomorphic vector fields, approximate holomorphic infinitesimal dilations, can be linearized. We use this result in Section 4 to prove the sufficient condition for weighted homogeneity.

Received October 10, 1994.

Research supported in part by NSF grants DMS 91-01113 and DMS 93-01345 and by NSF grant DMS-9022140 at MSRI. 


\section{Preliminaries}

Throughout this paper we will assume that $M$ is a real analytic real hypersurface through the origin in $\mathbf{C}^{n}$. We fix positive integers $m_{1}, \ldots, m_{n}$. The non-isotropic group of dilations determined by $\left(m_{1}, \ldots, m_{n}\right)$ is the group $\left\{\delta_{t}: t>0\right\}$ where

$$
\delta_{t}(z)=\left(t^{m_{1}} z_{1}, \ldots, t^{m_{n}} z_{n}\right)
$$

For a multiindex $A=\left(\alpha_{1}, \ldots, \alpha_{n}\right)$, we let $\|A\|=\sum m_{i} \alpha_{i}$ and $|A|=\sum \alpha_{i}$.

Definition 1.2. A function $h$ is homogeneous of weight $j$ if $h \circ \delta_{t}=t^{j} h$. A vector field $Y$ is homogeneous of weight $j$ if

$$
Y\left(f \circ \delta_{t}\right)=t^{-j}(Y f) \circ \delta_{t}
$$

We say a function $h=O(j)$ if every term in its Taylor expansion about 0 has weight $\geq j$. Similarly, if $Y$ is a vector field, we write $Y=O(j)$ if, when we expand the coefficients of $Y$ in a Taylor series about 0 , each term is a vector field of weight $\geq j$. If $Y$ is a vector field with $Y=O(j)$ and $h$ is a function with $h=O(k)$, it follows that $Y h=O(j+k)$.

We say a function is $O\left(|z|^{j}\right)$ if it vanishes to order $j$ at the origin. If $Y$ is a vector field, we write $Y=O\left(|z|^{j}\right)$ if every coefficient of $Y$ is $O\left(|z|^{j}\right)$.

Definition 1.3. A biholomorphic map $\phi$ is allowable if

$$
\phi(z)=\left(\phi_{1}(z), \ldots, \phi_{n}(z)\right), \quad \phi_{j}(z)=z_{j}+O\left(m_{j}+1\right) .
$$

Let

$$
Z_{0}=\sum_{j=1}^{n} m_{j} z_{j} \frac{\partial}{\partial z_{j}}
$$

and let

$$
Y_{0}=2 \operatorname{Re} Z_{0} .
$$

We call $Y_{0}$ an infinitesimal dilation because the one-parameter group generated by $Y_{0}$ is the group of non-isotropic dilations $\left\{\delta_{e^{t}}: t \in \mathbf{R}\right\}$. We call $Z_{0}$ a holomorphic infinitesimal dilation. If $f$ is a polynomial homogeneous of weight $k$ then $Y_{0} f=k f$.

A smooth vector field $X$ on $M$ is called an infinitesimal $C R$ automorphism of $M$ if the local one-parameter group it generates is a local group of CR automorphisms of $M$. Let aut $(M)$ denote the space of infinitesimal CR automorphisms of $M$ which are defined in a neighborhood of the 
origin. If $Z$ is a holomorphic vector field, i.e., a vector field of type $(1,0)$ with holomorphic coefficients, then the local group generated by $Y=\operatorname{Re} Z$ is a local group of holomorphic maps [KN, remarks preceding Proposition IX.2.10]. Hence, if $Y$ is a vector field defined on a neighborhood of the origin in $M$ and $Y=\left.(\operatorname{Re} Z)\right|_{M}$ for some holomorphic vector field $Z$, then $Y \in \operatorname{aut}(M)$. We denote the subspace of such infinitesimal CR automorphisms by $\operatorname{hol}(M)$.

Definition 1.4. A vector field $Y \in \operatorname{aut}(M)$ is an approximate infinitesimal dilation if $Y=Y_{0}+O(1)$. A holomorphic vector field $Z$ is an approximate holomorphic infinitesimal dilation if $Z=Z_{0}+O(1)$.

If $M$ is given by an equation of the form (0.1) where $P$ is a polynomial homogeneous with respect to the group $\left\{\delta_{t}\right\}$, then $Y_{0} \in \operatorname{hol}(M)$. We will see in Proposition 2.2 that if $M$ is equivalent to (0.1) via an allowable map, then $M$ has an approximate infinitesimal dilation in $\operatorname{hol}(M)$.

\section{Allowable maps}

In this section, we give some results concerning allowable biholomorphic maps. The first is that the inverse of an allowable map is allowable and that an allowable map takes an infinitesimal approximate dilation to an infinitesimal approximate dilation.

Lemma 2.1. Suppose $\phi$ is an allowable biholomorphic map. Then $\psi=$ $\phi^{-1}$ is allowable. If $Z$ is an approximate holomorphic dilation then $d \phi Z$ also is.

We omit the proof, which is straightforward. As a corollary, we have the following proposition.

Proposition 2.2. Suppose $M$ is a real analytic real hypersurface through the origin in $\mathbf{C}^{n}$ and that $M$ is equivalent to (0.1) via the restriction to $M$ of an allowable biholomorphic map $\phi$. Then there is an approximate infinitesimal dilation $Y \in \operatorname{hol}(M)$.

Proof. Let $Y=d \phi^{-1} Y_{0}$. Then $Y \in \operatorname{hol}(M)$. Because $\phi$ is allowable, by Lemma 2.1, $Y=Y_{0}+O(1)$.

Next we show that if $Z$ is an approximate holomorphic dilation then the linear part of $Z$ is diagonalizable.

Lemma 2.3. Suppose

$$
Z=\sum_{i} m_{i} z_{i} \frac{\partial}{\partial z_{i}}+O(1)
$$


Then there is an allowable linear transformation $z^{\prime}=S z$ such that

$$
d S Z=\sum_{i} m_{i} z_{i}^{\prime} \frac{\partial}{\partial z_{i}^{\prime}}+O(1) \cap O\left(\left|z^{\prime}\right|^{2}\right) .
$$

Proof. Write

$$
Z=\sum_{i, j} a_{i j} z_{j} \frac{\partial}{\partial z_{i}}+O\left(|z|^{2}\right)
$$

Let $A$ be the linear transformation with matrix $\left(a_{i j}\right)$. After reordering the coordinates if necessary, we may assume that $m_{i} \leq m_{i+1}$. Because $Z$ satisfies (2.4),

$$
a_{i j}=m_{i} \delta_{i j} \quad \text { if } \quad m_{i} \geq m_{j} .
$$

(So, in particular, $A$ is upper triangular.) We will find a linear transformation $T=\left(t_{i j}\right)$ such that

$$
t_{i j}=\delta_{i j} \quad \text { if } \quad m_{i} \geq m_{j}
$$

which diagonalizes $A$,

$$
T^{-1} A T z=D z
$$

where $D$ is the diagonal matrix with $d_{i i}=m_{i}$. Then by (2.7), $T$ is allowable. Hence, by Lemma 2.1, $S=T^{-1}$ is allowable. If $z^{\prime}=S z$ then, by (2.8) and Lemma 2.1, $d S Z$ satisfies (2.5).

By (2.6), (2.8) is equivalent to

$$
\left(m_{k}-m_{i}\right) t_{k i}=-\sum_{\left\{j: m_{k}<m_{j}\right\}} a_{k j} t_{j i}
$$

holding for $k=1, \ldots, n$. Because $m_{n} \geq m_{j}$ for all $j$, we can solve $\left(2.9_{n}\right)$ by taking

$$
t_{n i}=\left\{\begin{array}{ll}
1, & i=n \\
0, & i<n
\end{array} .\right.
$$

Suppose we have found $t_{j i}, j=k+1, \ldots, n, i=1, \ldots, n$, satisfying (2.7) and $\left(2.9_{j}\right)$. The right hand side of $\left(2.9_{k}\right)$ is known. Hence, $t_{k i}$ satisfies

$$
t_{k i}=\frac{-1}{m_{k}-m_{i}} \sum_{\left\{j: m_{k}<m_{j}\right\}} a_{k j} t_{j i}, \quad m_{k} \neq m_{i} .
$$


If $m_{j}>m_{k} \geq m_{i}$, then $j>k$ and, by $(2.7), t_{j i}=0$, so

$$
\sum_{\left\{j: m_{k}<m_{j}\right\}} a_{k j} t_{j i}=0, \quad m_{k} \geq m_{i}
$$

Hence, we can take

$$
t_{k i}=\delta_{k i}, \quad m_{k}=m_{i} .
$$

By $(2.10),(2.11)$ and $(2.12)$, we have $t_{j i}, j=k, \ldots, n, i=1, \ldots, n$, satisfying $(2.7)$ and $\left(2.9_{j}\right)$. Thus, by downward induction on $k$, we can find $T$ satisfying (2.7) and $\left(2.9_{k}\right)$, and hence (2.8).

\section{Linearization}

In this section, we prove the following linearization result.

Theorem 3.1. If $Z$ is an approximate holomorphic infinitesimal dilation, there is an allowable biholomorphic map $\phi$ such that $Z_{0}=d \phi Z$ is a holomorphic infinitesimal dilation.

By the Poincaré-Dulac Theorem [Po, Deuxième Partie, Théorème III], [Du] (see also [A, Theorem 24E]), there is a biholomorphic map taking $Z$ to a polynomial vector field in which all monomials of degree greater than 1 are resonant. It does not follow from this that $Z$ can be linearized, nor that the map may be taken to be allowable. However, Theorem 3.1 does follow from Poincaré's method of proof. One constructs the map as a formal power series and then uses a domination argument to prove convergence. Once one has a formal power series solution, one could invoke a more general result of Pliss [Pl] to obtain convergence. However, Pliss's proof uses successive approximations and is much more complicated.

Proof. By Lemma 2.3, we may assume that

$$
Z=\sum\left(m_{i} z_{i}+h_{i}(z)\right) \frac{\partial}{\partial z_{i}}, \quad h_{i} \in O\left(m_{i}+1\right) \cap O\left(|z|^{2}\right) .
$$

Let $z^{\prime}=\phi(z)=\left(\phi_{1}(z), \ldots, \phi_{n}(z)\right)$ be an allowable biholomorphic map,

$$
\phi_{j}(z)=z_{j}+f_{j}(z), \quad f_{j} \in O\left(m_{j}+1\right) .
$$

Then

$$
d \phi Z=\sum_{i, k}\left(m_{i} z_{i}+h_{i}(z)\right)\left(\delta_{i k}+\frac{\partial f_{k}}{\partial z_{i}}(z)\right) \frac{\partial}{\partial z_{k}^{\prime}}
$$


Hence, if

$$
d \phi Z=\sum m_{k} z_{k}^{\prime} \frac{\partial}{\partial z_{k}^{\prime}}
$$

equating coefficients of $\frac{\partial}{\partial z_{k}^{\prime}}$ in (3.2) and (3.3) gives

$$
\sum_{i}\left(m_{i} z_{i}+h_{i}(z)\right) \frac{\partial f_{k}}{\partial z_{i}}(z)=m_{k} f_{k}(z)-h_{k}(z)
$$

Because $h_{i} \in O\left(m_{i}+1\right) \cap O\left(|z|^{2}\right)$, we look for a solution $f_{k}$ of (3.4) with $f_{k} \in O\left(m_{k}+1\right) \cap O\left(|z|^{2}\right)$. We first find a formal power series solution. Write

$$
h_{j}=-\sum_{\substack{|\alpha| \geq 2 \\\|\alpha\|>m_{j}}} b_{j \alpha} z^{\alpha}, \quad f_{k}=\sum_{\alpha} a_{k \alpha} z^{\alpha} .
$$

Set $a_{k \alpha}=0$ if $|\alpha|=\|\alpha\| \leq 1$. Fix $N \geq 2$ and suppose we have found $\left\{a_{k \gamma}:\|\gamma\|<N\right\}$ with $a_{k \gamma}=0$ if $|\gamma| \leq 1$ or $\|\gamma\| \leq m_{k}$. Let $\|\alpha\|=N$. Substitute (3.5) into (3.4) and equate the coefficients of $z^{\alpha}$. This gives

$$
\left(\|\alpha\|-m_{k}\right) a_{k \alpha}=P_{\alpha}^{\prime}\left(b_{j \beta}, a_{k \gamma}:\|\beta\| \leq N,\|\gamma\| \leq N-1\right)
$$

where $P_{\alpha}^{\prime}$ is a polynomial with nonnegative coefficients and $P_{\alpha}^{\prime}(0)=0$. The terms on the right side only involve $a_{k \gamma}$ with $\|\gamma\| \leq N-1$ and hence are known. If $N \leq m_{k}$, the right side of (3.6) is 0 so we may take $a_{k \alpha}=0$. Thus, by induction, we see that

$$
a_{k \alpha}=P_{k \alpha}\left(b_{j \beta}:\|\beta\| \leq\|\alpha\|\right)
$$

where $P_{k \alpha}$ is a polynomial with nonnegative coefficients, and we can find a formal power series solution $f_{k}$ to (3.4) such that $a_{k \alpha}=0$ if $|\alpha| \leq 1$ or $\|\alpha\| \leq m_{k}$.

We can find $K^{\prime}>0$ and $r>0$ such that for all $j$ and $\alpha$,

$$
\left|b_{j \alpha}\right| \leq \frac{K^{\prime}}{r^{|\alpha|}}
$$

Let $K=\frac{K^{\prime}}{r^{2}}$ and set

$$
\mu(z)=\frac{K r\left(\sum_{j=1}^{n} z_{j}\right)^{2}}{r-\sum_{j=1}^{n} z_{j}}=K r^{2} \sum_{|\alpha| \geq 2} \frac{|\alpha| !}{\alpha ! r^{|\alpha|}} z^{\alpha} .
$$


Then the series for $\mu$ dominates the series for $h_{j}$ in $\{|z|<r\}$. Let $A$ denote the set of multiindices $\alpha$ such that $2 \leq|\alpha|<2 m_{k},\|\alpha\|>m_{k}$, and let

$$
c=\min \left(\left\{\frac{\|\alpha\|-m_{k}}{|\alpha|-1}: \alpha \in A\right\}, \frac{1}{2}\right) .
$$

Then $c>0$ because $A$ is finite. Suppose $f$ solves the equation

$$
\sum_{j=1}^{n} z_{j} \frac{\partial f}{\partial z_{j}}=\frac{\mu}{c}\left(\sum_{j=1}^{n} \frac{\partial f}{\partial z_{j}}+1\right)+f
$$

with $f(0)=0,\left.d f\right|_{0}=0$. Then the coefficient of $z^{\alpha}$ in the power series for $f$ is given by the right side of (3.7) with $b_{j \beta}$ replaced by $b_{\beta}=K r^{2} \frac{|\beta| !}{c \beta ! r|\beta|}$ and $\|\gamma\|-m_{k}$ replaced by $|\gamma|-1$. Hence, it is of the form

$$
\widetilde{P}_{\alpha}\left(b_{\beta}:|\beta| \leq|\alpha|\right)
$$

where each coefficient of $\widetilde{P}_{\alpha}$ is larger than the corresponding coefficient of $P_{k \alpha}$ because $c(|\alpha|-1)<\|\alpha\|-m_{k}$ if $|\alpha|>1,\|\alpha\|>m_{k}$. Hence, the series for $f$ majorizes the series for $f_{k}$.

To solve (3.8), we write $f(z)=\sum_{j=1}^{n} z_{j} v\left(\sum_{k=1}^{n} z_{k}\right)$ and solve the resulting regular ordinary differential equation for $v$ with $v(0)=0$. This gives

$$
f(z)=\frac{1}{n} \sum_{j=1}^{n} z_{j}\left(\left(1-(c+K n r) \sum_{k=1}^{n} \frac{z_{k}}{c r}\right)^{-K n r /(c+K n r)}-1\right)
$$

which solves (3.8) in a neighborhood of 0 with $f(0)=0,\left.d f\right|_{0}=0$ and has a convergent power series expansion in the neighborhood. Thus, because the series for $f$ dominates the one for $f_{k}$, the series for $f_{k}$ also converges.

\section{A criterion for homogeneity}

In this section, we prove our main result, Theorem 4.1, which gives a criterion for $M$ to be homogeneous. We also show, Proposition 4.3, that a homogeneous hypersurface is rigid.

Theorem 4.1. Let $M$ be an analytic real hypersurface through the origin in $\mathbf{C}^{n}$ and suppose there is an approximate infinitesimal dilation $Y \in \operatorname{hol}(M)$. Then $M$ is weighted homogeneous.

Proof. Because $Y \in \operatorname{hol}(M), Y=2 \operatorname{Re} Z$ where $Z$ is an approximate holomorphic infinitesimal dilation. By Theorem 3.1 there is an allowable biholomorphic change of coordinates $\widehat{z}=\phi(z)$ such that

$$
d \phi Z=\sum m_{j} \widehat{z}_{j} \frac{\partial}{\partial \widehat{z}_{j}} .
$$


After reordering the coordinates and replacing $\widehat{z}_{n}$ by $\imath \widehat{z}_{n}$ if necessary, we may assume that $M$ is given by an equation of the form

$$
\operatorname{Im} \widehat{z}_{n}-F\left(\widehat{z}^{\prime}, \bar{z}^{\prime}, \operatorname{Re} \widehat{z}_{n}\right)=0, \quad \widehat{z}^{\prime}=\left(\widehat{z}_{1}, \ldots \widehat{z}_{n-1}\right)
$$

Write $F=\sum F_{k}$ where $F_{k}$ is a polynomial homogeneous of weight $k$. Because $Y$ is tangent to $M$, applying $d \phi Y=Y_{0}$ to (4.2) gives

$$
\left.Y_{0}\left(\operatorname{Im} \widehat{z}_{n}-F\left(\widehat{z}^{\prime}, \overline{z^{\prime}}, \operatorname{Re} \widehat{z}_{n}\right)\right)\right|_{M}=-\sum_{k}\left(k-m_{n}\right) F_{k}\left(\widehat{z}^{\prime}, \widehat{z}^{\prime}, \operatorname{Re} \widehat{z}_{n}\right)=0
$$

Hence,

$$
F_{k}\left(\widehat{z}^{\prime}, \bar{z}^{\prime}, \operatorname{Re} \widehat{z}_{n}\right)=0, \quad k \neq m_{n}
$$

and $F$ is homogeneous of weight $m_{n}$. Thus, (4.2) is a polynomial equation homogeneous of weight $m_{n}$ and $M$ is weighted homogeneous.

Following the terminology of Baouendi, Rothschild and Treves [BRT], we call $M$ rigid if there are coordinates $\left(z_{1}, \ldots, z_{n-1}, w\right)$ such that $M$ is given by an equation of the form $\operatorname{Im} w=F(z, \bar{z})$ or, equivalently, if there is a transversal infinitesimal $\mathrm{CR}$ automorphism on $M$. Rigid hypersurfaces are called regular by Tanaka [T] and T-regular by D'Angelo [DA].

Proposition 4.3. If $M$ is weighted homogeneous then $M$ is rigid.

Proof. We can assume that, after a CR diffeomorphism if necessary, $M$ is given by a homogeneous polynomial equation of weight $m$. As in the proof of Theorem 4.1, we can assume that this equation is of the form

$$
\operatorname{Im} z_{n}=F\left(z^{\prime}, \overline{z^{\prime}}, \operatorname{Re} z_{n}\right), \quad z^{\prime}=\left(z_{1}, \ldots, z_{n-1}\right) .
$$

Then

$$
\operatorname{Im} z_{n}-a \operatorname{Re} z_{n}=p\left(z^{\prime}, \overline{z^{\prime}}\right)
$$

for some polynomial $p$ homogeneous of weight $m$ and some $a \in \mathbf{R}$. Let

$$
\widehat{z}_{j}=z_{j}, j=1 . ., n-1, \widehat{z}_{n}=\frac{1+a^{2}}{1+i a} z_{n} .
$$

In the new coordinates, the equation of $M$ is

$$
\operatorname{Im} \widehat{z}_{n}=p\left(\widehat{z}^{\prime}, \overline{\bar{z}^{\prime}}\right) .
$$

Hence $M$ is rigid. 


\section{References}

[A] V. I. Arnold, Geometrical methods in the theory of ordinary differential equations, Springer-Verlag, New York, 1983.

[BRT] M. S. Baouendi, L. P. Rothschild and F. Treves, CR structures with group action and extendability of CR functions, Inventiones Math. 82 (1985), 359-396.

[DA] J. P. D'Angelo, Defining equations for real analytic real hypersurfaces in $C^{n}$, Trans. A.M.S. 295 (1986), 71-84.

[Du] H. Dulac, Solutions d'un système d'équations différentielles dans le voisinage de valeurs singulières, Bull. Soc. Math. de France 40 (1912), 324-383.

[KN] S. Kobayashi and K. Nomizu, Foundations of differential geometry, II, Wiley Interscience, New York, 1969.

$[\mathrm{Pl}] \quad$ V. A. Pliss, On the reduction of an analytic system of differential equations to linear form, Differential Equations 1 (1965), 111-118.

[Po] H. Poincaré, Sur les propriétés des fonctions définies par les équations aux différences partielles, Première Thèse (1879); Euvres, I, IL-CXXIX, GauthierVillars, Paris, 1928.

[S1] N. K. Stanton, Rigid hypersurfaces in $C^{2}$, Proc. Symposia Pure Math., Vol. 52, Part 3, Amer. Math. Soc., Providence, 1991, pp. 347-354.

[S2] _ A normal form for rigid hypersurfaces in $C^{2}$, Amer. J. Math. 113 (1991), 877-910.

[S3] - Infinitesimal CR automorphisms of rigid hypersurfaces in $\mathbf{C}^{2}$, J. Geometric Analysis 1 (1991), 231-267.

[S4] Infinitesimal CR automorphisms of rigid hypersurfaces, Amer. J. Math. 117 (1995), 141-167.

[T] N. Tanaka, On the pseudo-conformal geometry of hypersurfaces of the space of $n$ complex variables, J. Math. Soc. Japan 14 (1962), 397-429.

Department of Mathematics, University of Notre Dame, Notre Dame, in 46556

E-mail address: Nancy.K.Stanton@nd.edu 